\title{
Publisher Correction: Mechanism of parkin activation by phosphorylation
}

Véronique Sauvé (1D), George Sung (1D, Naoto Soya (1D, Guennadi Kozlov, Nina Blaimschein, Lis Schwartz Miotto, Jean-François Trempe (i), Gergely L. Lukacs and Kalle Gehring (1)

Correction to: Nature Structural \& Molecular Biology https://doi.org/10.1038/s41594-018-0088-7, published online 2 July 2018.

In the version of this article initially published, RING2 in the schematic to the left in Fig. 1b was mislabeled as RING0. The error has been corrected in the HTML and PDF versions of the article.

Published online: 19 July 2018

https://doi.org/10.1038/s41594-018-0105-x 